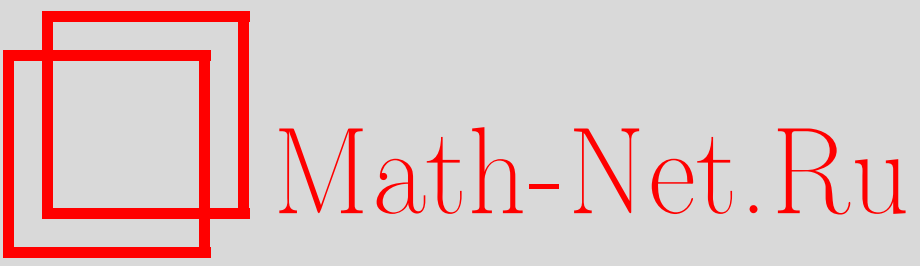

В. М. Бухштабер, К. Э. Фельдман, Формула сложения для первого полуцелого класса Понтрягина в комплексных кобордизмах, УМН, 1997, том 52, выпуск $6,151-152$

DOI: https://doi.org/10.4213/rm897

Использование Общероссийского математического портала Math-Net.Ru подразумевает, что вы прочитали и согласны с пользовательским соглашением

http://www. mathnet.ru/rus/agreement

Параметры загрузки:

IP : 3.85 .5 .30

26 апреля 2023 г., 16:25:58 


\title{
ФОРМУЛА СЛОЖЕНИЯ ДЛЯ ПЕРВОГО ПОЛУЦЕЛОГО КЛАССА ПОНТРЯГИНА В КОМПЛЕКСНЫХ КОБОРДИЗМАХ
}

\author{
В. М. БУХШТАБЕР, К. Э. ФЕЛЬДМАН
}

В работе [1] была предложена конструкция характеристических классов векторных расслоений на основе трансфера Беккера-Готтлиба [2], ассоциированного с грассманизацией этих расслоений. Это позволило для случая кобордизмов ввести аналоги классов Понтрягина вещественных векторных расслоений. Одним из ключевых результатов теории характеристических классов являются формулы сложения, выражающие классы суммы расслоений через классы слагаемых. В рамках конструкции [1] эти формулы являются следствием свойства локализации трансфера Беккера-Готтлиба. До последнего времени все формулы сложения для классов Понтрягина вещественных векторных расслоений были получены лишь с точностью до 2-примарных компонент. Основным результатом настоящей работы является полная теорема сложения для первого полуцелого класса Понтрягина в комплексных кобордизмах, которая опирается на новые результаты о локализации трансфера Беккера-Готтлиба, полученные в [3].

Дадим общее определение полуцелых классов Понтрягина в случае комплексных кобордизмов. Рассмотрим вещественное векторное расслоение $\xi(\operatorname{dim} \xi=n)$. Обозначим через $\mathbb{R} G_{k}^{n}(\xi)$ грассманизацию расслоения $\xi$ со слоем, являющимся вещественным многообразием Грассмана $\mathrm{k}$-мерных подпространств в $\mathbb{R}^{n}$. Пусть $\tau\left(p_{k}\right)$ трансфер Беккера-Готтлиба (в дальнейшем - просто трансфер) расслоения $\left(\mathbb{R} G_{k}^{n}(\xi), \mathbb{R G}_{k}^{n}, B, p\right)$, где $B$ - база расслоения $\xi$. Обозначим $k$-мерное тавтологическое векторное расслоение над $\mathbb{R} \mathrm{G}_{k}^{n}(\xi)$ через $\xi(k)$ и через $\chi(\eta)$ канонический Эйлеров класс комплексного векторного расслоения $\eta$ в комплексных кобордизмах.

ОПРЕДЕЛЕнИЕ 1. Пусть $\xi$ - $n$-мерное вещественное векторное расслоение. Классом Понтрягина $p_{k / 2}(\xi)$ в комплексных кобордизмах, $k=1,2, \ldots, n$, называется характеристический класс, задаваемый выражением:

$$
p_{k / 2}(\xi)=\tau\left(p_{k}\right)^{*} \chi(C \otimes \xi(k))
$$

Пусть $f(u, v)=u+v+\sum_{i, j \geqslant 1} \alpha_{i j} u^{i} v^{j}$ - формальная группа в комплексных кобордизмах [4] и $[u]_{2}=f(u, u)$. В комплексных кобордизмах естественно возникает ряд $a(u)=[u]_{2} / u$, как $\tau(\pi)^{*}(1)$ для двулистного накрытия $\pi: E \rightarrow B$, где $u$ - Эйлеров класс комплексификации одномерного вещественного векторного расслоения над $B$, ассоциированного с накрытием $\pi$. Введем ряд

$$
b(u, v)=\sum_{i, j \geqslant 1} \beta_{k l} u^{k} v^{l}=u v \frac{a(v)-a(u)}{u-v} .
$$

Теорема 1. Первый полуцелый класс Понтрягина в комплексных кобордизмах суммы двух вещественных векторных расслоений $\xi$ и

$$
p_{1 / 2}(\xi \oplus \zeta)=u+v+\sum_{k, l \geqslant 1} \beta_{k l} s_{l-1}(v) s_{k-1}(u),
$$

где $u=p_{1 / 2}(\xi), v=p_{1 / 2}(\zeta), s_{i}-$ операчии Ландвебера-Новикова [4].

Доказательство теоремы 1 использует следующие свойства трансфера и операций Ландвебера-Новикова (см. [2], [4], [5]):

1. Пусть $\tau(p)$ - трансфер расслоения $(E, F, B, p)$. Пусть $h^{*}(\cdot)$ - некоторая мультипликативная теория когомологий, и $x \in \widetilde{h}^{*}\left(B^{+}\right), y \in \widetilde{h}^{*}\left(E^{+}\right)$. Тогда $\tau(p)^{*}\left(p^{*}(x) y\right)=x \tau(p)^{*}(y)$.

2. Операции Ландвебера-Новикова коммутируют с гомоморфизмом, индуцированным в когомологиях трансфером расслоения: $s_{k} \tau(p)^{*}=\tau(p)^{*} s_{k}$.

3. Пусть $w \in \widetilde{U}^{2}\left(X^{+}\right)$реализуется как геометрический кобордизм, тогда $s_{k-1} w=w^{k}$.

Tеорема 2. Пусть $\xi_{1}-$ тавтологическое векторное расслоение над $\mathbb{R P}^{n}$. Тогда

$$
\operatorname{Ind}\left(\mathbb{R P}\left(\xi_{1} \oplus 1\right)\right)=\tau(p)^{*}(1)=-\sum_{i, j \geqslant 1} \alpha_{i j} w^{i+j-1},
$$

где $\tau\left(\right.$ р) - трансфер расслоения $\mathbb{R} \mathbb{P}\left(\xi_{1} \oplus 1\right), w=\chi\left(C \otimes \xi_{1}\right)$. 
Доказательство использует, что: пространство $\mathbb{R} \mathbb{P}\left(\xi_{1} \oplus 1\right)$ гомеоморфно двулистному накрытию над собой, ассоциированному с одномерным тавтологическим векторным расслоением над этим пространством; $p_{1 / 2}\left(\xi_{1} \oplus 1\right)=p_{1 / 2}\left(\xi_{1}\right)$ (см. [3]).

ДокаЗАтЕльство теОРемы 1 . Рассмотрим векторное расслоение $\xi$ над базой $B$. Пусть расслоение $\xi^{\perp}$ таково, что $\xi \oplus \xi^{\perp}$ - тривиальное расслоение. Рассмотрим отражение $\mu: T\left(\xi \oplus \xi^{\perp}\right) \rightarrow T\left(\xi \oplus \xi^{\perp}\right.$ ) относительно подрасслоения $\xi^{\perp}$ (через $T(\xi)$ мы обозначаем пространство Тома векторного расслоения $\xi$ ). Используя изоморфизм надстройки, получаем согласно [6], что индуцированное отображение $\mu^{*}: \widetilde{U}^{*}\left(B^{+}\right) \rightarrow \widetilde{U}^{*}\left(B^{+}\right)$есть умножение на элемент из $\widetilde{U}^{0}\left(B^{+}\right)$. Обозначим этот элемент через $\gamma(\xi)$. Из теоремы о локализации трансфера, полученной в работе [3], вытекает, что

$$
p_{1 / 2}(\xi \oplus \zeta)=p_{1 / 2}(\xi)+\tau(p)^{*}\left(\gamma\left(p^{*}(\xi) \otimes \zeta(1)^{*}\right) \cdot w\right),
$$

где $\tau(p)$ - трансфер расслоения $\mathbb{R P}(\zeta), w=\chi(C \otimes \zeta(1))$. Предположим сначала, что $\operatorname{dim} \xi=1$. Из теоремы 2 и свойств 1-3 получаем, что

$$
\gamma\left(\zeta \otimes \xi^{*}\right) \cdot u=u+\sum_{k, l \geqslant 1} \beta_{k l} u^{l} s_{k-1}(v),
$$

где $u=\chi(C \otimes \xi), v=p_{1 / 2}(\zeta)$. Рассмотрим случай произвольного $\xi$. Пусть $u=p_{1 / 2}(\xi)$. Имеем $p_{1 / 2}(\xi \oplus \zeta)=u+\tau(p)^{*}\left(\gamma\left(p^{*}(\xi) \otimes \zeta(1)^{*}\right) w\right)$

$$
=u+\tau(p)^{*}\left(w+\sum_{k, l \geqslant 1} \beta_{k l} w^{l} p^{*} s_{k-1}(u)\right)=u+v+\sum_{k, l \geqslant 1} \beta_{k l} s_{l-1}(v) s_{k-1}(u) .
$$

СледствиЕ 1. В комплексной $K$-теории имеет место равенство

$$
p_{1 / 2}(\xi \oplus \zeta)=f\left(p_{1 / 2}(\xi), p_{1 / 2}(\zeta)\right)
$$

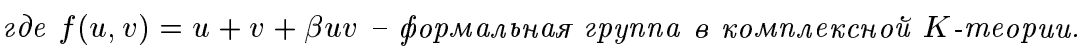

СледствиЕ 2. Если вещественное векторное расслоение $\eta$ допускает комплексную структуру, то $p_{1 / 2}(\eta)=0$.

СлеДСтвиЕ 3. Первый полущелый класс Потрягина вещественного векторного расслоения над конечным клеточным комплексом 2-примарен.

Теорема 3. Характеристический класс $p_{1 / 2}$ задает отображение

$$
p_{1 / 2}: K O(X) \rightarrow U^{2}(X),
$$

на образе которого имеется ассочиативная операция сложения

$$
x \oplus y=x+y+\sum_{k, l \geqslant 1} \beta_{k l} s_{l-1}(y) s_{k-1}(x) .
$$

Обратим внимание, что в случае комплексных кобордизмов эта операция не сводится ни к какой формальной группе.

\section{СПИСОК ЛИТЕРАТУРЫ}

[1] Бухштабер В. М. // Изв. АН СССР. Сер. матем. 1978. Т. 42. № 1. С. 130-184. [2] Becker J.C., Gottlieb D. H. // Topology. 1975. V. 14. № 1. P. 1-12. [3] Бухштабер B. M., Фельдман К.Э. Локализация трансфера и характеристические классы Понтрягина (в печати). [4] Новиков С. П. // Изв. АН СССР. Сер. матем. 1967. Т. 31. № 4. С. 855-951. [5] Бухштабер В. М. // Итоги науки и техн. Совр. пробл. матем. Фундамент. направл. Т. 10. М.: ВИНИТИ, 1978. С. 5-178. [6] Dold А. // Invent. Math. 1974. V. 25. Р. 281-287.

Московский государственный университет им. М.В. Ломоносова
Принято редколлегией 20.10 .1997 